\title{
Targeting the mutant p53 secretome
}

\author{
Kartik Sehgal ${ }^{1,2}$ and David A. Barbie ${ }^{1,2}$ \\ 'Department of Medical Oncology, Dana-Farber Cancer Institute, Boston, Massachusetts, USA. ${ }^{2 H a r v a r d}$ Medical School, Boston, Massachusetts, USA.
}

\begin{abstract}
While $\mathrm{p} 53$ is the most highly mutated and perhaps best studied tumor suppressor protein related to cancer, it remains refractory to targeted therapeutic strategies. In this issue of the JCI, Tan and colleagues investigated the mechanistic basis of the mutant p53 secretome in preclinical models of lung adenocarcinoma. The authors uncovered miR-34a as a regulator of a conventional protein secretion axis, which is mediated by three proteins: the Golgi reassembly and stacking protein 55 kDa (GRASP55), basic leucine zipper nuclear factor 1, and myosin IIA. Inhibition of CRASP55 in TP53-deficient lung adenocarcinoma suppressed protumorigenic secretion of osteopontin/secreted phosphoprotein 1 and insulin-like growth factor binding protein 2 and reduced tumor growth and metastases in mice as well as in patient-derived xenografts. These results provide a therapeutic opportunity to target downstream effects of p53 loss.
\end{abstract}

\section{Protumorigenic extracellular factors}

The cancer secretome comprises multiple macromolecules (proteins, lipids, microRNAs, messenger RNA) secreted by cancer cells and associated stromal cells in the tumor microenvironment (1). It is markedly altered compared with the normal tissue secretome and contributes to tumor invasion and metastasis through maintenance of cancer stem cells, generation of a protumorigenic microenvironment, and development of premetastatic niches. Specific extracellular factors facilitate cell-tocell interactions and extracellular matrix remodeling and are released through tightly regulated conventional protein secretion (CPS) or unconventional protein secretion (UPS) pathways (2). While CPS accomplishes extracellular release of factors with signal peptides and/or transmembrane domains through the classical ER-Golgi apparatus, UPS is a stress-mediated process that bypasses the Golgi organelles. Recent efforts have focused on usage of secretome analyses for cancer biomarker development and therapeutic targeting.

Genetic (e.g., TP53, $c-M y c$, and PTEN) and epigenetic (e.g., microRNA) alterations in cancer cells and their interactions with the tumor microenvironment mediate the dysregulation of secreted factors (1). Understandably, the cancer secretome associated with mutant TP53 (encoding p53 protein) receives widespread attention, since this tumor suppressor is the most frequently altered gene in cancer (3). While disruption of this aptly termed "guardian of the genome" dysregulates the normal cellular response to DNA damage, including DNA repair, cell cycle arrest, apoptosis, and senescence, p53 also alters the production and secretion of protumorigenic extracellular factors (4-6) (Figure 1). These

\section{Related Article: https://doi.org/10.1172/JCI137186}

Conflict of interest: DAB is an inventor on pending patents Microfluidic cell culture of patient-derived tumor cell spheroids (US10472599B2) and Methods for evaluating tumor cell spheroids using 3D microfluidic cell culture (US20190112666A); is a consultant for N-of-One and Tango Therapeutics; has received honoraria from Loxo Oncology, Merck, H3 Biomedicine/ Eisai, and Madalon Consulting; has received research grants from Bristol Myers Squibb, Novartis, Eli Lilly and Company, and Gilead Sciences Inc.; and is cofounder of and on the scientific advisory board of Xsphera Biosciences Inc.

Copyright: @ 2021, American Society for Clinical Investigation.

Reference information: / Clin Invest. 2021;131(1):e143945. https://doi.org/10.1172/JCl143945.

non-cell-autonomous effects of mutant p53 can remodel the extracellular matrix, alter proinflammatory cytokine secretion, modulate tumor-stromal crosstalk, and promote extracellular acidification (6). For example, mutant p53 was found to induce a proinvasive secretome in H1299 lung adenocarcinoma (LUAD) cells by recruiting p63 as a molecular chaperone, which subsequently altered expression of target genes (7). p53 also affects protein stability and/or secretion. Reactivation of WT p53 function in LN-Z308 glioma cells induced differential expression of 60 proteins in the conditioned media, none of which were regulated at the transcriptional level (8). Notably, modulating the downstream effects of p53 loss provides a therapeutic opportunity for this as-yet-undruggable target.

\section{The mutant $\mathrm{p} 53$ secretome}

In this issue of the JCI, Tan and colleagues sought to identify therapeutic vulnerabilities in p53-deficient LUAD by investigating the mechanistic basis of the mutant p53 secretome (9). The researchers employed multiple preclinical LUAD models, including TP53 WT cells (A549), TP53-null cells (H1299, CALU-1), and TP53-missense mutant cells with (murine 344SQ, 344P) and without (H2122) gain-of-function properties. Most in vitro experiments were performed in A549, H1299, and H2122, while in vivo experiments employed 344P, 344SQ, H1299, and A549, as well as TP53-mutant patient-derived xenografts (PDXs) (9).

Tan and coauthors first analyzed data from The Cancer Genome Atlas and identified increased expression of Golgi reassembly stacking protein 55 (GRASP55) in LUAD and other cancers harboring TP53 mutations and alterations in other cancer driver genes (9). GRASP55 not only is involved in Golgi structure formation and CPS but also has been implicated in UPS pathways, including autophagosome/ endosome-based secretion and the ER stress-mediated Golgi bypass pathway (2, 10). Preclinical LUAD models confirmed that loss of WT p53 function was associated with increased levels of GRASP55 


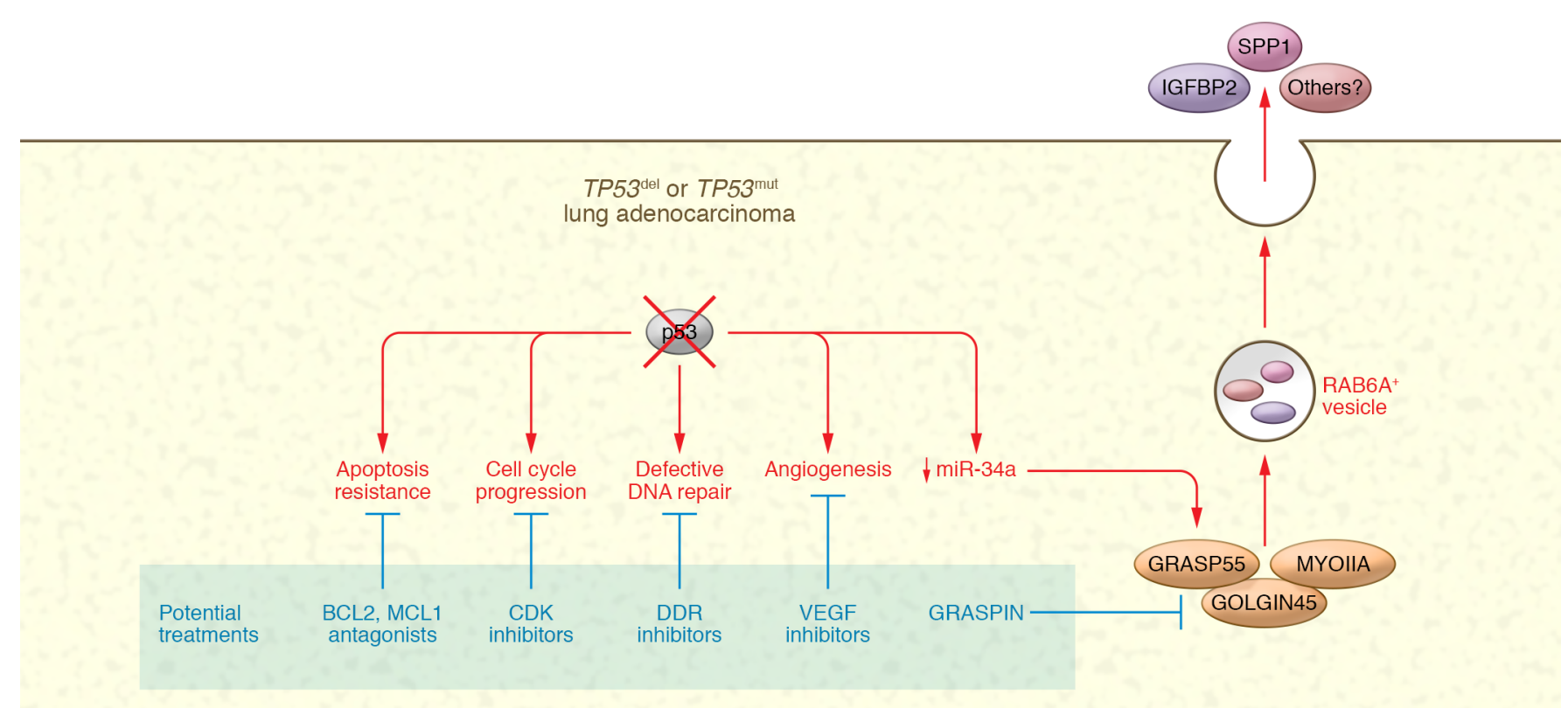

Figure 1. Targeting downstream effects of mutant p53. Mutant p53 dysregulates multiple downstream pathways in cancer cells, promoting resistance to apoptosis, bypass of cell cycle checkpoints, defective DNA repair, and angiogenesis, as well as a secretion axis that is regulated by suppression of miR-34a expression. The output of this secretome involves RAB6A+ vesicle-mediated extracellular release of insulin-like growth factor binding protein-2 (IGFBP2) and osteopontin/secreted phosphoprotein 1 (osteopontin/SPP-1) through Golgi reassembly-stacking protein 55/ GOLGIN45/myosin IIA (GRASP55/GOLGIN45/MYOIIA). As compared with theoretical targeting of other downstream pathways, GRASPIN, a small molecule inhibitor of GRASP55, prevents GRASP55 from binding with GOLGIN45 and consequently can inhibit this secretory axis (9). BCL2, B cell lymphoma 2; CDK, cyclin-dependent kinase; DDR, DNA damage response; MCL1, myeloid cell leukemia 1.

(9). The mutant p53-driven upregulation of GRASP55 was indirectly mediated via transcriptional silencing of miR-34a upon p53 inactivation; miR-34a, which itself has tumor suppressive properties (11), was shown to bind to the $3^{\prime}$-untranslated region of GRASP55. The authors further demonstrated selective dependence of TP53-mutant LUAD cells on GRASP55 for cell proliferation, colony formation, migration, and invasion, as compared with TP53-proficient LUAD cells (9). These data were supported by in vivo studies demonstrating a role of GRASP55 in driving tumor growth and metastases in 344P and 344SQ models. Through depletion/ neutralization and rescue experiments, the authors identified osteopontin/SPP1 and IGFBP2 as the major extracellular factors contributing to the effects of GRASP55 on cancer cell growth, migration, and invasion (9). Consistently, both SPP-1 and IGFBP2 have been previously implicated in tumorigenesis and immunosuppression (12-15).

Further support for the hypothesis that mutant p53/miR-34a/GRASP55 axis promotes downstream SPP-1 and IGFBP2 secretion was that $\mathrm{p} 53$ failed to transcriptionally regulate both genes (9), a finding that is also consistent with data from a previous proteomic analysis (8). Additional mechanistic investigations revealed that the Golgi phosphoprotein 3-independent (GOLPH3-independent) CPS pathway, involving GRASP55, GOLGIN45 (G45), MYOIIA, and RAB6A, regulated SPP-1 and IGFBP2 secretion. Depletion of GRASP55, G45, or MYOIIA reduced SPP1 and IGFBP2 levels in post-Golgi vesicles, but not in Golgi-enriched cellular fractions, with subsequent functional impairment of colony formation, migration, and invasion. Additionally, GRASP55 promoted the stability of $\mathrm{G} 45$ protein by inhibiting its ubiquitin-mediated degradation, and the silencing of miR-34a also upregulated MYOIIA, establishing multiple overlapping regulatory control mechanisms in the context of LUAD. Notably, GRASP55 depletion also decreased the secretion of proinflammatory and proangiogenic cytokines, interleukin-6, VEGF-A, and CXCL5/LIX, suggesting an additional impact on the tumor immune microenvironment. Together, these data support the presence of a miR-34a-dependent GRASP55/G45/MYOIIA CPS regulatory axis activated downstream of mutant $\mathrm{p} 53$, as well as potential therapeutic targets in LUAD.

The small molecule inhibitor GRASPIN can target the GRASP55 docking N-terminal PSD-95/discs large/ZO-1 domain to compete with binding partners, such as G45. TP53-mutant LUAD cells exhibited $50 \%$ lower half maximal inhibitory concentration $\left(\mathrm{IC}_{50}\right)$ values following GRASPIN treatment compared with TP53-proficient cells, further supporting the differential vulnerability to GRASP55 depletion in this p53-deficient context. GRASPIN treatment, expectedly, reduced secretion of both SPP1 and IGFBP2. Furthermore, concomitant repletion of both factors led to more effective, albeit incomplete, rescue of protumorigenic cancer cell properties, suggesting a role for GRASP55-regulated secretion of other protumorigenic factors, or potential off-target effects of GRASPIN treatment. Tan and coauthors further demonstrated that treatment with GRASPIN reduced tumor growth and metastatic burden in multiple in vivo LUAD preclinical models, 
including PDXs. Interestingly, within the short time frame of these in vivo mouse studies, no substantial toxicities, such as weight loss, were noticed (9).

\section{Conclusions and clinical implications}

Tan et al. shed fundamental insights on a GOLPH3-independent CPS axis mediated by GRASP55/G45/MYOIIA, which is in turn regulated by $\mathrm{p} 53 / \mathrm{miR}-34 \mathrm{a}$ in LUAD (9). It is, however, important to note that multiple p53-independent mechanisms of regulation of miR-34a have also been described (16). While multiple preclinical LUAD (including KRAS WT and mutant) models were utilized, for several key experiments, the authors relied on RNA interference, which is associated with greater off-target effects as compared with CRISPR knockout. Furthermore, though preliminary analysis of TP53-mutant breast and ovarian cancer cell models in this study (9) showed similar antitumorigenic effects of GRASP55 depletion, tissue specificity of action also needs further investigation. Variations in transcription factors, chromatin landscape, and co-occurring genetic alterations may ultimately determine respective importance of various regulatory secretory axes in other cancer histological subtypes (5). For example, a mutant p53/HIF1 $\alpha /$ miR-30d axis has been recently identified in syngeneic breast cancer models, which potentiates tubule-vesiculation of the Golgi apparatus and induces a prometastatic secretome (17). Last, while GRASPIN treatment showed encouraging in vivo results with negligible side effects, the time frame utilized in current studies was short (9), and mouse models often fail to predict human toxicity. By virtue of GRASP55 inhibition, GRASPIN also has the potential to affect multiple cellular pathways, such as lipid metabolism and interleukin- $1 \beta$ signaling
$(10,18,19)$, and needs more comprehensive evaluation of its on-target and off-target toxicities in vivo.

TP53 has remained an elusive target for anticancer therapeutic development because of the myriad ways its dysfunction promotes tumor development and metastases through cell-autonomous and noncell-autonomous effects (3). As promising therapeutics emerge for other previously undruggable targets, such as oncogenic $K R A S$, there is a renewed interest in a multipronged approach to overcome the effects of the most common genetic alteration in cancer cells. Previous efforts to inhibit downstream mutant p53 pathways, such as PDGFR- $\beta$ signaling by imatinib, showed promise preclinically in pancreatic adenocarcinoma (20), but have not yet translated to efficacy in the clinic. Other potential approaches to exploit p53-regulated cancer cell dependencies include apoptosis modulation, cyclin-dependent kinase inhibition, synthetic lethality of DDR inhibitors, angiogenesis inhibition, and as described here, blocking the mutant $\mathrm{p} 53$ cancer secretome downstream of GRASP55 (Figure 1). However, further delineation of whether potent/specific GRASP55 inhibition or degradation exhibits robust preclinical efficacy will dictate whether this turns out to be the opportunity to overcome another undruggable target.

Address correspondence to: David A. Barbie, Dana-Farber Cancer Institute, 450 Brookline Avenue, Boston, Massachusetts 02215, USA. Phone: 617.632.6036; Email: david_barbie@dfci.harvard.edu.

1. Paltridge JL, et al. The secretome in cancer progression. Biochim Biophys Acta. 2013;1834(11):2233-2241.

2. Rabouille C. Pathways of unconventional protein secretion. Trends Cell Biol. 2017;27(3):230-240.

3. Tang Q, et al. Mutant p53 on the path to metastasis. Trends Cancer. 2020;6(1):62-73.
4. Vogelstein B, et al. Surfing the p53 network. Nature. 2000;408(6810):307-310.

5. Pavlakis E, Stiewe T. p53's extended reach: the mutant $\mathrm{p} 53$ secretome. Biomolecules. 2020;10(2):307.

6. Cordani M, et al. Mutant p53 proteins alter cancer cell secretome and tumour microenvironment: involvement in cancer invasion and metastasis. Cancer Lett. 2016;376(2):303-309.

7. Neilsen PM, et al. Mutant p53 uses p63 as a molecular chaperone to alter gene expression and induce a pro-invasive secretome. Oncotarget. 2011;2(12):1203-1217.

8. Khwaja FW, et al. Proteomic identification of the wt-p53-regulated tumor cell secretome. Oncogene. 2006;25(58):7650-7661.

9. Tan X, et al. A protumorigenic secretory pathway activated by $\mathrm{p} 53$ deficiency in lung adenocarcinoma. J Clin Invest. 2021;131(1):e137186.

10. Ahat E, et al. New insights into the Golgi stacking proteins. Front Cell Dev Biol. 2019;7:131.

11. Adams BD, et al. The tumor-suppressive and potential therapeutic functions of miR-34a in epithelial carcinomas. Expert Opin Ther Targets. 2016;20(6):737-753.

12. Zhao H, et al. The role of osteopontin in the progression of solid organ tumour. Cell Death Dis. 2018;9(3):356.

13. Klement JD, et al. An osteopontin/CD44 immune checkpoint controls CD8+ T cell activation and tumor immune evasion. JClin Invest. 2018;128(12):5549-5560.

14. Yao X, et al. IGF-binding protein 2 is a candidate target of therapeutic potential in cancer. Tumour Biol. 2016;37(2):1451-1459.

15. Liu Y, et al. IGFBP2 promotes immunosuppression associated with its mesenchymal induction and Fc $\gamma$ RIIB phosphorylation in glioblastoma. PLoS One. 2019;14(9):e0222999.

16. Slabáková E, et al. Alternative mechanisms of miR-34a regulation in cancer. Cell Death Dis. 2017;8(10):e3100.

17. Capaci V, et al. Mutant p53 induces Golgi tubulo-vesiculation driving a prometastatic secretome. Nat Commun. 2020;11(1):3945.

18. Kim J, et al. Grasp55\%- mice display impaired fat absorption and resistance to high-fat diet-induced obesity. Nat Commun. 2020;11(1):1418

19. Chiritoiu M, et al. GRASP55 and UPR control interleukin-1 $\beta$ aggregation and secretion. Dev Cell. 2019;49(1):145-155.e4.

20. Weissmueller S, et al. Mutant p53 drives pancreatic cancer metastasis through cell-autonomous PDGF receptor $\beta$ signaling. Cell. 2014;157(2):382-394. 